\title{
Efectos del Trabajo Infantil en la Salud del Menor Trabajador
}

\author{
LEONARDO BRICEÑO AYALA ${ }^{1}$ y ÁNGELA M PINZÓN RONDÓN² \\ ${ }^{1}$ Médico. Especialista en Salud Ocupacional. Facultad de Medicina de la Universidad del \\ Rosario. Teléfono: 3474570 Ext. 211 o 240. Fax: 3474570 Ext. 210. Dirección: Calle 63D \# \\ 24-31, Bogotá, Colombia. \\ E-mail: lbriceno@urosario.edu.co \\ ${ }^{2}$ Medico. Master Public Health. Facultad de Medicina de la Universidad del Rosario. Telé- \\ fono: 3474570 Ext. 236 o 331. Fax: 6101848 o 3474570 Ext. 210. Dirección: Calle 63D \# 24- \\ 31, Bogotá, Colombia. \\ E-mail: ampinzon@urosario.edu.co
}

Recibido 17 Agosto 2004/Enviado para Modificación 21 Septiembre 2004/Aceptado 17 Octubre 2004

\section{RESUMEN}

Objetivos Revisamos el tema del trabajo infantil, en tres aspectos: 1. Las características de los niños que los hacen vulnerables ante el trabajo; 2 . Las enfermedades y accidentes relacionados con el trabajo infantil, y 3 . Las lesiones asociadas con diversas ocupaciones de menores, como agricultura, construcción, manufactura, textiles, industria del cuero, ventas en calle y trabajo en plazas de mercado.

Métodos Se consultaron las bases de datos Medline, EBSCO, PROQUEST e HINARI, y material de la Organización Internacional del Trabajo, la Academia Americana de Pediatría y la Asociación Americana de Psicología.

Resultados La información acerca de riesgos ocupacionales en niños es muy escasa comparativamente con la de adultos. Se encontró que los niños tienen características que los hacen especialmente vulnerables a los riesgos laborales y no existen estándares claros para protegerlos.

Palabras Claves: Trabajo de menores, revisión, factores de riesgo, vulnerabilidad, ocupación (fuente: DeCS, BIREME).

\section{ABSTRACT}

Effects of child labor upon the health of the child worker

Objectives This article presents a review of the evidence on child labor, in three aspects: 1 . The characteristics of children which make them vulnerable to labor; 2 . The diseases and accidents related with child labor, and 3 . The injuries associated with the childs' occupations, such as agriculture, construction, manufacture, textiles, leather industry, street sales and sales in marketplaces. 
Methods The Medline, EBSCO, PROQUEST and HINARI databases were searched, and material from the International Labor Organization, the American Academy of Pediatrics and the American Association of Psychology was reviewed.

Results Information regarding occupational risk in children is poor as compared with information in adults. Children have characteristics which make them particularly vulnerable to occupational risks and there are no clear standards to protect them.

Key Words: Child labor, review, risk factors, vulnerability, occupation (source: MeSH, NLM).

$\mathrm{M}$ ás de 246 millones de niños entre los 5 y los 17 años se encuentran actualmente trabajando en todo el mundo y 180 millones se encuentran sometidos a las peores formas de trabajo infantil, como lo son la explotación sexual, las tareas domésticas, el trabajo en minas, en fábricas de fuegos pirotécnicos y en los conflictos armados. Latinoamérica tiene al 16\% de los niños que laboran en estas condiciones. En países industrializados, no obstante que el trabajo infantil es menos común, el fenómeno esta creciendo, particularmente en los países del este de Europa (1). En Colombia existen aproximadamente 1567847 niños trabajadores (2) y se presenta el fenómeno incluso en menores de 5 años $(3,4)$. A pesar de la magnitud del problema, los estudios sobre el tema en referencia son escasos.

Las causas del trabajo infantil son múltiples y entre estas podemos destacar: la pobreza, que constituye un círculo vicioso al asociarse con el trabajo infantil (5), la creencia errada que hace pensar que el trabajo infantil es bueno y la carencia de políticas socioeconómicas en contra del trabajo infantil.

Desafortunadamente en el ámbito legal no existe un límite claro entre lo permitido y lo prohibido en trabajo infantil (6). Casi todas las personas han tenido algún tipo de trabajo antes de cumplir los 18 años y esta experiencia puede traer cosas positivas $(7,8)$. Sin embargo, existe hoy en el mundo una gran cantidad de menores que ven truncado su futuro por comenzar a trabajar prematuramente.

Diferentes investigaciones científicas han demostrado como el trabajo afecta el desarrollo de los niños y jóvenes, los expone a sufrir accidentes y los predispone a desarrollar múltiples enfermedades. Los niños son más vulnerables que los adultos por la etapa de desarrollo en la cual se encuentran. Además algunos niños y jóvenes en el trabajo se encuentran bajo presión 
porque desconocen el ambiente laboral, necesitan conservar su trabajo y deben aportar al sustento diario de su familia (1). Los niños son tan vulnerables a los factores de riesgo laborales que incluso algunas investigaciones han mostrado como la exposición laboral de los padres influye en su salud al afectarlos en etapas muy tempranas de desarrollo (9 - 11).

La literatura científica disponible relacionada con la salud y el trabajo se caracteriza por ser prolija en estudios realizados en adultos. Es así como los límites de exposición máxima frente a contaminantes ambientales están relativamente bien definidos para adultos sanos. Pero los puntos de referencia en poblaciones infantiles son supremamente vagos, inespecíficos o sencillamente no existen.

Este artículo muestra las características que hacen a los niños seres altamente vulnerables ante el trabajo y presenta una revisión de los estudios relacionados con las enfermedades y accidentes asociados a los factores de riesgo laborales y ocupaciones específicas.

\section{METODOLOGÍA}

El presente artículo contiene una revisión del tema del trabajo infantil en la bibliografía académica reciente. Se revisaron los siguientes temas: 1. las características de los niños que los hacen vulnerables ante el trabajo; 2. las enfermedades y accidentes relacionados con el trabajo infantil, y 3. las lesiones asociadas con diversas ocupaciones de menores.

Las fuentes de información exploradas en lo pertinente a los temas planteados fueron: La base de datos bibliográfica Medline, y las bases de datos textuales EBSCO, PROQUEST e HINARI. Se consultaron también las bases de datos, la literatura científica y las revisiones sistemáticas de la Organización Internacional del Trabajo, la Academia Americana de Pediatría y la Asociación Americana de Psicología. Finalmente se escogieron documentos relacionados con trabajo infantil de la Administración de Salud y Seguridad Ocupacional (Osha) y del Instituto Nacional de Seguridad y Salud Ocupacional (Niosh).

La información encontrada se enmarcó y complementó con textos básicos de Salud Ocupacional e informes nacionales sobre trabajo infantil del Departamento Administrativo Nacional de Estadística. 
Los descriptores utilizados y las estrategias de búsqueda variaron dependiendo de la base de datos explorada de la siguiente manera:

Para Medline se usaron los siguientes descriptores: Chilhood, occupational risks, child labour and health, children health and environment, children development and occupation y endocrine disruptors in children. Se realizaron búsquedas sin límites de tiempo. Para Niosh y Osha se escogieron los documentos en el buscador alfabético de cada una de estas agencias.

Niosh: Children, Childhood agricultural injury topic page, Youth workers y Young workers safety and health topic page.

Osha: Youth y Youth in agriculture e-tool.

Para OIT se revisaron los documentos a texto completo disponibles en la página del Programa InFocus sobre trabajo infantil y en el Sistema de Información Regional sobre Trabajo Infantil (Sirti).

La metodología aplicada en esta revisión no implicó una asociación por plausibilidad biológica sino una revisión de los estudios existentes. En otras palabras, aunque es evidente que las mismas lesiones que se encuentran en los adultos expuestos se pueden presentar en los niños que tengan exposiciones similares, esta revisión se centró en buscar las lesiones que efectivamente se han estudiado en trabajo de menores.

No se evaluó el nivel de evidencia de las fuentes revisadas.

\section{RESULTADOS}

Vulnerabilidad biopsicosocial del menor trabajador

El desarrollo infantil, el cual hace parte del ciclo vital de desarrollo, incluye el período prenatal, la infancia, la niñez temprana y la niñez intermedia. La Tabla 1 resume la vulnerabilidad de los niños en cada una de estas etapas. Entre más pequeño sea un niño, más vulnerable será al trabajo. La herencia y el ambiente interactúan en cada una de estas etapas.

Los niños al estar en un proceso dinámico de crecimiento y desarrollo tienen características que los hacen más propensos que los adultos a los factores de riesgo en el trabajo (12). Cada órgano y/o sistema presenta características fisiológicas especiales durante su desarrollo. 
Tabla 1. Exposición ambiental y vulnerabilidad de acuerdo con los estados del desarrollo de la preconcepción a la adolescencia (6)

\begin{tabular}{|c|c|c|c|}
\hline $\begin{array}{c}\text { Estado del desa- } \\
\text { rrollo }\end{array}$ & $\begin{array}{c}\text { Características del } \\
\text { desarrollo }\end{array}$ & Exposición & Vulnerabilidad \\
\hline Preconcepción & $\begin{array}{l}\text { Exposición } \\
\text { Gonadal }\end{array}$ & $\begin{array}{l}\text { Todos los factores } \\
\text { de riesgo }\end{array}$ & Genotoxicidad \\
\hline Embarazo & $\begin{array}{l}\text { Alto consumo } \\
\text { calórico. } \\
\text { Placenta } \\
\text { permeable }\end{array}$ & $\begin{array}{l}\text { Todos los } \\
\text { contaminantes } \\
\text { ambientales }\end{array}$ & $\begin{array}{l}\text { Teratogenicidad } \\
\text { debida al desarrollo } \\
\text { embrionario de } \\
\text { diferentes órganos } \\
\text { y aparatos }\end{array}$ \\
\hline Primeros 3 años & $\begin{array}{l}\text { Exploración oral } \\
\text { Comienzo de la } \\
\text { ambulación. Dieta } \\
\text { estereotipada }\end{array}$ & $\begin{array}{l}\text { Comida } \\
\text { Aire } \\
\text { Piso. }\end{array}$ & $\begin{array}{l}\text { Daño potencial a } \\
\text { cerebro (sinapsis) y } \\
\text { pulmones (alvéolos } \\
\text { en desarrollo). } \\
\text { Sensibilización } \\
\text { alérgica. } \\
\text { Accidentes }\end{array}$ \\
\hline $\begin{array}{l}\text { Edad preescolar y } \\
\text { escolar }\end{array}$ & $\begin{array}{l}\text { Mayor } \\
\text { independencia } \\
\text { Trabajo infantil }\end{array}$ & $\begin{array}{l}\text { Comida } \\
\text { Aire } \\
\text { Exposición } \\
\text { ocupacional }\end{array}$ & $\begin{array}{l}\text { Daño potencial al } \\
\text { cerebro (formación } \\
\text { de sinapsis } \\
\text { específicas, } \\
\text { dendritas) y } \\
\text { pulmones } \\
\text { (Expansión de } \\
\text { volumen). } \\
\text { Accidentes }\end{array}$ \\
\hline Adolescencia & $\begin{array}{l}\text { Pubertad } \\
\text { Crecimiento } \\
\text { Conductas de } \\
\text { Riesgo } \\
\text { Trabajo infantil }\end{array}$ & $\begin{array}{l}\text { Comida } \\
\text { Aire } \\
\text { Agua } \\
\text { Exposición } \\
\text { ocupacional }\end{array}$ & $\begin{array}{l}\text { Daño potencial al } \\
\text { cerebro (formación } \\
\text { de sinapsis } \\
\text { específicas, } \\
\text { dendritas) y } \\
\text { pulmones } \\
\text { (Expansión de } \\
\text { volumen). } \\
\text { Desarrollo puberal } \\
\text { Accidentes }\end{array}$ \\
\hline
\end{tabular}

El sistema osteomuscular de los niños se caracteriza por tener huesos con menos elasticidad y por tanto menor fuerza y capacidad de soporte de carga. Esta característica hace que los niños estén más propensos a sufrir diferentes desordenes osteomusculares al transportar manualmente cargas, realizar trabajos repetitivos o al adoptar posturas por tiempos prolongados o posiciones incómodas. Igualmente los niños presentan crecimiento y desarrollo de las 
extremidades superiores e inferiores, lo que incide en la precisión de sus movimientos y los predispone a sufrir accidentes cuando trabajan con equipos o en situaciones peligrosas.

Los órganos vitales cambian de diámetro, volumen y madurez. Los trabajos con equipo y maquinaria que generen vibraciones, con carga física o mental intensa, con exposición crónica a polvos, humos, vapores o gases, en espacios confinados, en profundidades o cámaras, con exposiciones a temperaturas extremas, o con exposición a productos peligrosos ocasionan mayor alteración a los órganos en desarrollo y esto es especialmente importante para el sistema nervioso que tiene una limitada capacidad de regeneración $(13,14)$. Además, el desarrollo del sistema neurológico es la base fundamental donde se cimientan los demás desarrollos $(15,16)$. Estas alteraciones van desde pequeños cambios neurológicos, cardiacos y respiratorios hasta cáncer e insuficiencias importantes que incluso pueden llevar a la muerte.

El sistema reproductivo se está desarrollando y ocupaciones con exposición a altas temperaturas, campos electromagnéticos o con productos peligrosos pueden causar alteraciones en su crecimiento y desarrollo que llevan a infertilidad y cáncer (17).

En cuanto al desarrollo y la madurez psicosocial, los niños se encuentran en proceso de evolución de su personalidad y por esto trabajos con peligro de violencia, abuso o adicción los predisponen, aun más que los adultos, a sufrir trastornos psicosociales y además los hace más vulnerables a situaciones laborales con alta exigencia mental y física.

El niño es el gestor de su propio desarrollo y son muy importantes su autoestima el desarrollo de su creatividad, y su felicidad. Los niños y niñas trabajadoras con frecuencia trabajan en actividades que son explotadoras, peligrosas y degradantes. Muy a menudo son maltratados, abusados y abandonados por sus patrones. Estos niños están especialmente propensos a sufrir alteraciones psicosociales.

Además el trabajo infantil interfiere con el desarrollo social y académico de los niños. El trabajo les quita tiempo para desarrollar otras actividades como jugar, aprender y compartir en familia. Varias investigaciones han mostrado como los niños trabajadores tienen una muy alta deserción escolar $\mathrm{y}$ un alto retraso escolar $(2-4,8)$

Teniendo en cuenta que desde una perspectiva biopsicosocial, se enfatiza en la importancia del desarrollo del niño y su relación con el concepto de 
salud, el trabajo infantil va en contra de los derechos fundamentales de los niños.

Las interacciones negativas entre las condiciones de trabajo y los factores humanos pueden conducir a perturbaciones emocionales, problemas de comportamiento y cambios bioquímicos y neuro-hormonales que presentan riesgos adicionales de enfermedades mentales y físicas. Pueden también preverse efectos nocivos para la satisfacción y el rendimiento en el trabajo.

Por otro lado, los niños están proporcionalmente más expuestos por unidad de peso corporal a las toxinas ambientales que los adultos. Por lo cual la evaluación del riesgo se debe realizar de manera especial $(18,19)$. La mayor vulnerabilidad de los niños a los químicos esta dada por factores (20) como el hecho de que los niños proporcionalmente comen, respiran y toman más agua por kilo de peso corporal, y sus vías metabólicas son inmaduras (21). Además los niños tienen más años de vida futura y así más tiempo para desarrollar enfermedades iniciadas por exposiciones tempranas $(22,23)$.

Accidentes y enfermedades relacionadas con trabajo infantil

Accidentes. Los niños y jóvenes son nuevos en el trabajo, inexpertos, no se atreven a preguntar sobre su trabajo ni a hacer exigencias y no conocen sus derechos como trabajadores (5), esto los hace especialmente vulnerables a sufrir accidentes en el trabajo.

En países donde existen estadísticas más precisas de trabajo de menores, como los Estados Unidos, 200000 trabajadores entre los 14 y 17 años tienen lesiones relacionadas con el trabajo anualmente, de los cuales 64000 requieren tratamiento de urgencias y 70 mueren (24). Entre 1992 y 1997 se presentaron 403 muertes de menores trabajadores. Estas muertes ocurrieron primordialmente en trabajos de agricultura (40\%), ventas al por menor (22 $\%)$, construcción (13\%), y servicios ( $8 \%$ ). Una tercera parte de las muertes ocurrieron en negocios familiares y el $60 \%$ de éstas tuvieron un vehículo o maquinaria involucrados, el $20 \%$ fueron debidas a asaltos o hechos violentos y el $11 \%$ a enfermedad por sustancias tóxicas (24). Estos porcentajes son muy similares a las muertes laborales en adultos.

Para prevenir los accidentes de los niños trabajadores se requieren acciones por parte de los empleadores, los padres, las agencias reguladoras, la comunidad en general y los niños por sí mismos (5). Y para prevenir las 
muertes se recomienda que los menores de edad no manejen maquinaria peligrosa y tengan un horario laboral reducido.

En Colombia no poseemos datos de lesiones labores debidas el trabajo en menores, sin embargo es de suponer que las actividades económicas donde hay enfermedad por equipos y herramientas peligrosos estos deben producirse aunque no se registren o se informen.

Enfermedades Ocupacionales. Algunas enfermedades que se han visto rela cionadas con exposición a factores laborales son:

Asma: La aparición de asma a temprana edad ha sido asociada a humo de madera, petróleo, carbón, exhostos, herbicidas, pesticidas, polvo de granjas, granjas con cosechas y granjas de animales (25)

Cáncer Infantil: La leucemia linfoblástica aguda (26) y el cáncer cerebral (27) son lesiones que típicamente se han encontrado más en niños expuestos a agentes tóxicos.

Desórdenes del Neurodesarrollo (28): De profunda incidencia en su vida futura, se ha detectado el desarrollo de discapacidades del aprendizaje, dislexia, retardo mental, desorden de déficit de la atención y autismo (29-33). En la actualidad se estudian los efectos neurosicológicos de los contaminantes y por tanto se determina el estado de variables como la atención y la concentración, la función sensorial, el control motor, las relaciones espaciales, la coordinación visomotora, la memoria y el afecto (34).

Disrupción Endocrina: Los disruptores endocrinos son químicos que tienen la capacidad de interferir son el sistema de señalización hormonal del cuerpo (35). Sus efectos incluyen cáncer hepático y mamario por la exposición a dioxinas $(36,37)$, leucemia linfoblástica aguda por la exposición a plaguicidas -DDT, endosulfan-, ftalatos, bisfenol, disminución de la fertilidad (38) por la exposición a furanos (39), desórdenes del neurodesarrollo (33) y reducción de la inteligencia por la exposición a bifenilos policlorinados (40), diabetes, disfunción tiroidea $(41,42)$, disfunción del desarrollo sexual (4349) y en la conducta del rol sexual relacionada a genero por la exposición a bifenilos policlorinados (50), así como acelerado comienzo de la pubertad en niñas expuestas a bifenilos polibrominados. Estos últimos son usados como retardantes del fuego y en ciertos pesticidas como el dicofol, el pentaclorofenol, el dinoseb y el bromoxil $(51,52)$.

Intoxicaciones $(16,53)$ : No se conoce la dosis a la que no hacen daño los metales pesados en niños. La exposición a Plomo en niños a temprana edad a niveles 4 veces menores de los permitidos para los adultos puede generar retardo del desarrollo mental temprano (54), disminución del coeficiente de inteligencia, discapacidades de la lectura y la escritura, déficit de la atención, lenguaje y problemas persistentes de la conducta (55-60). La exposición a 
plomo también se ha asociado a alteraciones en el tallo cerebral (61). Quienes trabajan expuestos a petróleo, pinturas, baterías (62), plomerías, cerámicas de vidrio y radiadores (63) están expuestos a esta sustancia. Se han detectado problemas neurológicos que se expresan como agresividad, hiperactividad, distractilidad, desorganización y baja memoria (64). Igualmente anemia microcitica (65) tal como en los adultos expuestos. A nivel renal se han documentado cambios glomerulares y tubulointersticiales con glicosuria, proteinuria falla renal crónica e hipertensión (66).

La exposición a Metilmercurio ha mostrado efectos adversos en el neurodesarrollo (67). La exposición a monóxido de carbono causa síntomas similares a la influenza, fatiga, dolor de cabeza, mareos, nausea, vómitos, alteraciones cognitivas y taquicardia (68). El dióxido de nitrógeno y dióxido de azufre puede causar irritación aguda mucocutánea y efectos respiratorios. A nivel crónico la exposición a bajos niveles ha sido relacionada con asma e irritación respiratoria (69). La exposición a partículas del humo de madera puede resultar en irritación e inflamación del tracto respiratorio manifestadas con rinitis, tos, sibilancias o empeoramiento del asma (70,71). Otros tóxicos que se ha visto causan alteraciones en los niños son Tolueno, Bifenilos Policlorinados, Radiación Ionizantes, Plaguicidas y Manganeso (72,73). Es importante recalcar que estas son solo las sustancias tóxicas que se han estudiado en niños y que a medida que los estudios continúen la lista se va a incrementar.

Ocupaciones, factores de riesgo y lesiones en menores trabajadores

A continuación se presentan las lesiones asociadas a diversas ocupaciones de menores.

Agricultura

Es la ocupación infantil más común en el mundo (74), y se inicia desde temprana edad (75). Los niños generalmente son trabajadores no calificados, y frecuentemente deben elaborar y aplicar la mezcla para utilizar fertilizantes de cultivos, agroquímicos y plaguicidas. Dentro de los factores de riesgo están:

- Trabajo con equipo peligroso: Los tractores y en especial el mal manejo de los mismos (76), son responsables de la mayoría de los accidentes (77). Igualmente, los implementos de corte, equipo con partes rápida- 
mente giratorias, vagones pesados (78), picas, molinos, y combinadores de granos, son otra causa importante.

- Exposición a Plaguicidas u otros tóxicos (78): Inhalación, contacto, ingestión (79). Altos niveles de uso de agroquímicos (80).

- Pobre Higiene: Falta de agua potable e instalaciones de limpieza de manos y baños (8)

- Horarios Inapropiados: Desde la madrugada o desde tarde en la noche con horarios semanales y diarios extensos

- Alto Trabajo Físico: Levantamiento de cargas, y posturas inapropiadas. Temperaturas Extremas: Exposición a calor, bajo consumo de agua.

- Ruido: Por encima de limites permisibles en la operación de tractores (81). Exposición a animales: Mordeduras de insectos, serpientes y otros (82). Estampidas de animales o animales domésticos peligrosos, como el trabajo de parto o con animales recién nacidos (82).

- Trabajo en estructuras altas y en espacios confinados: riesgo de caídas y deprivación de oxígeno o una atmósfera tóxica (82).

Los efectos en la salud que se presentan son:

- Accidentes: La agricultura presenta la más alta tasa de accidentes en menores trabajadores (82); en Estados Unidos varía entre el 0,46 y el 8,4 $\%(83,84)$. Los niños son especialmente vulnerables $(84)$. Presenta una mortalidad calculada de entre 13,7 a 16,8 por 100000 (85). En Estados Unidos el $40 \%$ de las muertes de menores trabajadores se presentan en agricultura (86). En España se estima que los niños menores de 15 años sufren entre el 14 y el $24 \%$ de los accidentes fatales en agricultura $(8,77)$

- Intoxicación por Plaguicidas. Especialmente compromiso del sistema nervioso autonómico (87) y el sistema nervioso central. La muerte generalmente se produce por falla respiratoria. La exposición crónica produce efectos a largo plazo de carácter neurológico, y tumores con actividad hormonal (87).

- Lesiones por Calor: Especialmente en países tropicales (89).

- Infecciones: Por enfermedades parasitarias.

- Otros: pérdida auditiva, aumento de la presión sistólica (90) y asma ocupacional (91).

- Desórdenes musculoesqueléticos. Desordenes psicológicos: Depresión, abuso de sustancias y acoso sexual. 


\section{Construcción}

En una encuesta llevada a cabo en 26 países (18), el 1,9 \% de los niños laboraban en esta actividad. Los factores de riesgo en esta actividad están dados por trabajo expuesto a líneas de energía y a maquinaria peligrosa (86). Agentes como el ruido, asbesto, plomo, cemento y sus componentes, solventes y otras sustancias químicas son particularmente relevantes $(1,92)$. También se exponen a transporte manual de cargas, trabajos repetitivos, y posturas incómodas.

En cuanto los efectos en la salud, el 25,6 \% de los niños trabajadores de la construcción reportan algún tipo de accidente o enfermedad (18). Estudios realizados en adolescentes muestran una mortalidad del 15 por 100000 (93), la cual obedece principalmente a caídas y electrocuciones. En varios estudios la frecuencia de accidentes no fatales varía entre 0,33 y $25,7 \%$ (87). Hay una alta prevalencia de dolor musculoesqueletico, lumbar y en articulaciones $(77,94)$.

\section{Manufactura}

Se calcula que hasta el $8,3 \%$ de los niños trabajadores puede laborar en la manufactura en los países en desarrollo (18). Existe una amplia variedad de tareas a desarrollar en el campo de la manufactura por parte de los niños. Las labores con mayor frecuencia en los países en desarrollo son las fábricas de ladrillo, vidrio, fósforos y tapetes (95). Los efectos en la salud más importantes son los accidentes sobre todo por el uso de maquinaria (96) y las enfermedades musculoesqueléticas (77).

Textiles $(82,89,94)$

Los factores de riesgo son: Jornadas extensas, maquinaria peligrosa, ruido, cargas elevadas de polvo de algodón y otras fibras, solventes, tinturas, y bicromato de potasio. Los efectos más comunes son problemas musculoesqueléticos, accidentalidad, pérdida auditiva, bisinosis, dermatosis, cáncer de vejiga e intoxicaciones.

Industria del cuero

Los factores de riego son fuego, electrocución, maquinas peligrosas, postura estática sedente, exposición a sustancias químicas -Tinturas, Formaldehido, Pegantes (Pentaclorofenato de sodio, p-cloro-m-cresol), Solventes-(97). Y los efectos en la salud asociadas han sido las lesiones musculoesqueléticas (98) y las neurológicas (99). 
Vendedores ambulantes y otros trabajos en la calle

Las labores consisten en ventas en las carreteras y calles y los factores de riesgo son peligro de violencia (hostigamiento psicológico en el trabajo), accidentes de tránsito, jornadas extenuantes y trabajo nocturno (4). Igualmente polución ambiental, combustión de combustible fósil e incluye los contaminantes primarios como oxido sulfúrico, oxido de nitrógeno, aerosoles ácidos secundarios y otras partículas, como también oxidantes (99).

Los efectos en la salud encontrados son predisposición a adquirir conductas disociales, abuso y hostigamiento sexual, drogadicción, alteraciones a la funcionalidad social y familiar, lesiones físicas, heridas, golpes, enfermedad respiratoria como la neumonía (100), dermatitis y conjuntivitis.

Trabajo en plazas de mercado

Sus funciones son cargar y descargar mercancías de vehículos. Los factores de riesgo son transporte manual de cargas, trabajos repetitivos, posturas y posiciones incómodas, jornadas prolongadas y extenuantes y trabajo nocturno. Igualmente exposición prolongada a bajas temperaturas en cámaras frigoríficas y a ambientes con cambios extremos de temperatura. Los efectos en la salud reportados son: enfermedades músculo esqueléticas, fatiga, accidentes, alteraciones psicosociales, insuficiencia cardiaca y respiratoria, aumento de la frecuencia cardiaca y tensión arterial, lesión en piel y tejido subcutáneo, vasoconstricción, daño a vasos sanguíneos, insuficiencia cardiaca, fatiga, mareos e hipotermia.

\section{CONCLUSIONES}

Los niños se encuentran en una etapa del desarrollo tanto físico como psicológico muy importante y tanto fisiológica como psicológicamente son inmaduros, por esta razón son mucho más vulnerables que los adultos a la exposición a cualquier factor de riesgo. Teniendo en cuenta que los ambientes laborales presentan una gran cantidad de factores de riesgo, los niños en lo posible no deben ser expuestos a estos ambientes. La salud es un derecho de los niños y se debe propender por su promoción y por la prevención de enfermedades.

La cantidad de menores trabajadores es alarmante y debe disminuirse para lo cual el IPEC, SIMPOC (Programa de Información Estadística y Seguimiento en Materia de Trabajo Infantil) y la Organización Internacional del Trabajo, proponen un plan con siete componentes básicos: Estadística, armonización legislativa, creación de sistemas integrales de monitoreo e ins- 
pección, fortalecimiento de los agentes sociales, focalización de los programas sociales públicos, diversos programas de acción y creación de observatorios nacionales que miden la reducción del trabajo infantil. Igualmente, son necesarias medidas estructurales a largo plazo como el incremento sostenido en la inversión en la educación publica, aplicar una cobertura mayor a la protección social y que las políticas nacionales aseguren un trabajo decente para los adultos (6).

Mientras se logra la erradicación total del trabajo infantil es conveniente mantener a los niños trabajadores alejados de los factores de riesgo más significativos, como son maquinaria, tóxicos y los mismos agentes no estudiados aplicando márgenes de seguridad efectivos *

\section{REFERENCIAS}

1. Forastieri V. Children at work: Health and safety risks. Geneva: International Labour Office; 1997.

2. Departamento Administrativo Nacional de Estadística, Programa Internacional para la Erradicación del Trabajo Infantil Sudamérica. Encuesta Nacional de Trabajo Infantil. 1 ed. Bogotá (Colombia): DANE - OIT; 2001.

3. Florez C, Méndez C. Niños, Niñas y Jóvenes Trabajadores en Colombia 1996 Regina. Bogotá: OIT/IPEC-SIRTI; 1998. p. 168.

4. Pinzón AM, Briceño L, Gomez AI, Latorre C. Trabajo Infantil en las Calles de Bogotá. Rev. Cienc. Salud. 2003; 1(2):151-163.

5. Mardis AL, Pratt SG. Nonfatal injuries to young workers in the retail trades and services industries in 1998. J Occup. Environ Med. 2003; 45(3):316-323.

6. Barling J, Kelloway EK, editores. Young Workers: Varieties of Experiences. Washington D.C.: American Psychological Association; 1999.

7. Resnick MD, Bearman PS, Blum RW, Bauman KE, Harris KM, Jones J, et al. Protecting adolescents from harm. Findings from the National Longitudinal Study on Adolescent Health. JAMA. 1997; Sep 10;278(10):823-832.

8. Committee on the Health and Safety Implications of Child Labor, Board on Children, Youth, and Families, Commission on Behavioral and Social Sciences and Education National Research Council, Institute of Medicine. Protecting Youth at Work: Health, Safety, and Development of Working Children and Adolescents in the United States. Washington DC: National Academy Press; 1998.

9. Ronda E, Regidor E. Higher birth weight and lower prevalence of low birth weight in children of agricultural workers than in those of workers in other occupations. J Occup Environ Med. 2003; 45(1):34-40.

10. Meinert R, Kaletsch U, Kaatsch P, Schuz J, Michaelis J. Meinert R, Kalestsh U, Kaatsch P. Associations between childhood cancer and ionizing radiation: 
results of a population-based case-control study in Germany. Cancer Epidemiol Biomarkers Prev. 1999; 8(9):793-799.

11.Hum L, Kreiger N, Finkelstein MM. The relationship between parental occupation and bone cancer risk in offspring. Int J Epidemiol. 1998; 27(5):766771.

12. DeBaun MR, Gurney JG. Environmental exposure and cancer in children. A conceptual framework for the pediatrician. Pediatr Clin North Am. 2001; 48(5):1215-1221.

13. Rice D, Barone S Jr. Critical periods of vulnerability for the developing nervous system: evidence from humans and animal models. Environ Health Perspect. 2000;108 Suppl 3:511-533.

14. Rodier PM. Developing brain as a target of toxicity. Environ Health Perspect. 1995;103 Suppl 6:73-76.

15. Schettler T. Toxic threats to neurologic development of children. Environ Health Perspect. 2001;109 Suppl 6:813-816.

16. Cullen M, Mattison DR. Disorders of reproduction and development en Rosentock L. Textbook of occupational and environmental medicine. Philadelphia: Saunders; 1994.

17. Chia SE, Tay SK. Occupational risk for male infertility: a case-control study of 218 infertile and 227 fertile men. J Occup Environ Med. 2001; 43(11):946951.

18. Landrigan PJ, Kimmel CA, Correa A, Eskenazi B. Children's health and the environment: public health issues and challenges for risk assessment. Environ Health Perspect. 2004;112(2):257-265.

19. Renwick AG, Dorne JL, Walton K. An analysis of the need for an additional uncertainty factor for infants and children. Regul. Toxicol. Pharmacol. 2000; 31(3):286-296.

20. National Academy of Sciences (NAS). Pesticides in the Diets of Infants and Children. Washington, DC: National Academy Press; 1993.

21. Chemtob, S. Basic pharmacological principles. Fetal and neonatal physiology. Philadelphia W.B: Saunders; 1992.

22. Gray LE Jr, Ostby J, Furr J, Price M, Veeramachaneni DN, Parks L. Perinatal exposure to the phthalates DEHP, BBP, and DINP, but not DEP, DMP, or DOTP, alters sexual differentiation of the male rat. Toxicol. Sci. 2000; 58(2):350-365.

23. Ekbom A, Hsieh CC, Lipworth L, Adami HQ, Trichopoulos D. Intrauterine environment and breast cancer risk in women: a population-based study. J. Natl Cancer Inst. 1997; 89(1):71-76.

24. Nacional Institute for occupational safety and health. Resquest for assistance in preventing deaths and injuries of adolescent workers. Cincinnati: NIOSH; 1995.

25. Salam MT, Li Y, Langholz B, Gilliland FD. Early-Life Environmental Risk Factors for Asthma: Findings from the Children's Health Study. Environ Health Perspect. 2004;112:760-765.

26. Robison LL, Buckley JD, Bunin G. Assessment of environmental and genetic factors in the etiology of childhood cancers: the Children's Cancer Group 
epidemiology program. Environ Health Perspect. 1995;103 (suppl 6): S111S116.

27. Schechter CB. Brain and other central nervous system cancers: recent trends in incidence and mortality. J. Natl. Cancer Inst. 1999; 91(23):2050-2051.

28. National Research Council (NRC). Scientific Frontiers in Developmental Toxicology and Risk Assessment. Washington, DC: National Academy Press; 2000.

29. National Academy of Sciences (NAS) Environmental Neurotoxicology. Washington, DC: National Academy Press; 1992.

30. Campbell CG, Seidler FJ, Slotkin TA. Chlorpyrifos interferes with cell development in rat brain regions. Brain Res. Bull. 1997; 43(2):179-189.

31. Eskenazi B, Bradman A, Castorina R. Exposures of children to organophosphate pesticides and their potential adverse health effects. Environ Health Perspect. 1999;107 Suppl 3: 409-419.

32. Whitney KD, Seidler FJ, Slotkin TA. Developmental neurotoxicity of chlorpyrifos: cellular mechanisms. Toxicol. Appl. Pharmacol. 1995; 134(1):53-62.

33. Jacobson JL, Jacobson SW. Intellectual impairment in children exposed to polychlorinated biphenyls in utero. N Engl J Med. 1996; 335(11):783-789.

34. Fiedler N, Feldman RG, Jacobson J, Rahill A, Wetherell A. The assessment of neurobehavioral toxicity: SGOMSEC joint report. Environ Health Perspect. 1996;104 (Suppl 2):179-191.

35. Harrison PT. Endocrine disruptors and human health. BMJ. 2001; 323 (7325):1317-1318

36. Birnbaum LS, Fenton SE. Cancer and Developmental Exposure to Endocrine Disruptors. Environ Health Perspect. 2003; 111(4): 389-394.

37. Kogevinas M, Becher H, Benn T, Bertazzi P, Boffetta P, Bueno-de-Mesquita H, et al. Cancer mortality in workers exposed to phenoxy herbicides, chlorophenols, and dioxins. An expanded and updated international cohort study. Am J Epidemiol. 1997; 145(12):1061-1075.

38. Peterson RE, Theobald HM, Kimmel GL. Developmental and reproductive toxicity of dioxins and related compounds: cross species comparisons. Crit. Rev. Toxicol. 1993;23(3):283-335.

39. Guo YL, Hsu PC, Hsu CC, Lambert GH. Semen quality after prenatal exposure to polychlorinated biphenyls and dibenzofurans. Lancet. 2000;356(9237):1240-1241.

40. Winneke G. Endpoints of developmental neurotoxicity in environmentally exposed children. Toxicol. Lett. 1995; 77(1-3):127-136.

41. Longnecker MP, Daniels JL. Environmental contaminants as etiologic factors for diabetes. Environ Health Perspect. 2001; 109 Suppl 6:871-876.

42. Hauser P, McMillin JM, Bhatara VS. Resistance to thyroid hormone: implications for neurodevelopmental research on the effects of thyroid hormone disruptors. Toxicol. and Health.1998;14(1-2):85-101.

43. Euling S, Kimmel CA. Developmental stage sensitivity and mode of action information for endocrine disrupting chemicals. Sci. Total Environ. 2001; 274(1-3):103-113. 
44. Guillette LJ Jr, Gross TS, Masson GR, Matter JM, Percival HF, Woodward AR. Developmental abnormalities of the gonad and abnormal sex hormone concentrations in juvenile alligators from contaminated and control lakes in Florida. Environ Health Perspect. 1994; 102(8):680-688.

45. Blanck HM, Marcus M, Tolbert PE, Rubin C, Henderson AK, Hertzberg VS, et al. Age at menarche and tanner stage in girls exposed in utero and postnatally to polybrominated biphenyl. Epidemiology. 2000; 11(6):641-647.

46. Birnbaum LS. The mechanism of dioxin toxicity: relationship to risk assessment. Environ Health Perspect. 1994; 102 Suppl 9:157-167.

47. Selevan SG, Kimmel CA, Mendola P. Identifying critical windows of exposure for children's health. Environ Health Perspect. 2000; 108 (suppl 3):S451455.

48. Wu T, Buck GM, Mendola P. Blood lead levels and sexual maturation in U.S. girls: The Third National Health and Nutrition Examination Study, 19881994. Environ Health Perspect. 2003; 111(5):737-741.

49. Colborn T, Dumanoski D, Myers JP. Our Stolen Future: Are We Threatening Our Fertility, Intelligence, and Survival? A Scientific Detective Story. New York:Dutton; 1996.

50. Weisglas-Kuperus N, Patandin S, Berbers GA, Sas TC, Mulder PG, Sauer PJ, et al. Immunologic effects of background exposure to polychlorinated biphenyls and dioxins in Dutch preschool children. Environ Health Perspect. 2000;108 (12):1203-1207.

51. Darnerud P, Eriksen G, Johannesson T, Larsen P, Viluksela M. Polybrominated diphenyl ethers: occurrence, dietary exposure, and toxicity. Environ Health Perspect. 2001;109 (suppl 1):49-68.

52. Needham L, Hill R, Ashley D, Pirkle J, Sampson E. The priority toxicant reference range study: interim report. Environ Health Perspect. 1995;103 (suppl 3):89-94.

53. Schettler T. Toxic Threats to Neurologic Development of Children. Environ Health Perspect. 2001;109 Suppl 6:813-816.

54. Lanphear BP, Dietrich K, Auinger P, Cox C. Cognitive deficits associated with blood lead concentrations $<10 \mathrm{microg} / \mathrm{dL}$ in US children and adolescents. Public Health Rep. 2000;115(6):521-529.

55. Bellinger D, Leviton A, Waternaux C, Needleman H, Rabinowitz M. Longitudinal analyses of prenatal and postnatal lead exposure and early cognitive development. N. Engl. J. Med. 1987;316 (17):1037-1043.

56. Needleman HL, McFarland C, Ness RB, Fienberg SE, Tobin MJ. Bone lead levels in adjudicated delinquents- a case control study. Neurotoxicol Teratol. 2002;24(6):711-717.

57. Nyland LJ, Grimmer K. Is undergraduate physiotherapy study a risk factor for low back pain? A prevalence study of LBP in physiotherapy students. BMC Musculoskelet Disord. 2003;4(1):22.

58. Repko JD, Corum CR. Critical review and evaluation of the neurobiological and behavioral skeletal of inorganic lead absortion. CRC Crit. Rev. Toxicol. 1979; 6(2):135-187. 
59. Anderson AC, Pueschel SM, Linakis JG. Phatophisiology of lead poisoning. en: Pueschel SM, Linakis JG. Anderson AC, editors: Lead Posinining in childhood, Paul H Brookes Publishing Co Inc: Baltimore; 1996.

60. Dietrich KN, Succop PA, Bornschein RL, Krafft KM, Berger O, Hammond PB, et al. Lead exposure and neurobehavioral development in later infancy. Environ Health Perspect. 1990 Nov;89:13-9.

61. Counter SA, Brainstem Neural Conduction Biomarkers in Lead-Exposed Children of Andean Lead-Glaze Workers. J Occup Environ Med. 2002 Sep;44(9):855-64.

62. Sanborn MD, Albensohn, A, Campbell M, Weir E. Identifying in Managing Adverse Environmental Health Effects: 3 Lead Exposure. CMAJ. 2002 May 14;166(10):1287-92. Review.

63. Whittaker S. Lead Exposure in Radiator Repair Workers: A Survey of Washington State Radiator Repair Shops and Review of Occupational Lead Exposure Registry Data. J Occup Environ Med. 2003 Jul;45(7):724-33.

64. Campo A, Rambla C, de la Rosa J. Perfil Psiquiátrico de niños, niñas y Adolescente Maltratados (de la calle). Pediatría. 2000;35(2):96-100.

65. Agency for toxic Substances and Disease Registry. Case Studies in environmental medicine: lead toxicity. Atlanta (GA) 2000. [Internet] Disponible en: http://www.atsdr.cdc.gov/HRC/CSEM. Consultado en marzo 2 del 2004.

66. Kim RA. Longuitudinal study of low level lead exposure and imparment of renal function. JAMA 1996; 275(1):1177-1181.

67. United States Environmental Protection Agency (EPA). Chronic Reference Dose for Methylmercury ( $\mathrm{MeHg}$ ) Integrated Risk Information System. Washington, DC:U.S. Environmental Protection Agency; 2001.

68. Lambert WE, Samet JM. Indoor air pollution. In: Harber P, Schenker MM, Balmes JR (ed.) Occupational and Environmental Respiratory Disease. St Louis, MO:Mosby; 1996. p.784.

69. Morrow PE. Toxicological data on NOx: an overview. J. Toxicol. Environ. Health. 1984; 13(2-3):205-227.

70. Morris K, Morgenlander M, Coulehan JL, Gahagen S, Arena VC, Morganlander $\mathrm{M}$. Wood-burning stoves and lower respiratory tract infection in American Indian children. Am. J. Dis. Child. 1990;144(1):105-108.

71. Robin LF, Less PS, Winget M, Steinhoff M, Moulton LH, Santosham M, et al. Wood-burning stoves and lower respiratory illnesses in Navajo children. Pediatr. Infect. Dis J. 1996;15(10):859-865.

72. International Joint Commission United States and Canada. Great Lakes Water Quality Agreement; 1972. Renewed 1978.

73. McCarthy S. Threat of NAFTA case kills Canada's MMT ban. The Globe and Mail; July 20, 1998.

74. Fassa AG, Facchini LA, Dall'agnol MM, Christiani DC. Child labor and health: problems and perspectives. Int. J. Occup. Environ. Health. 2000;(1):55-62.

75. International Labor Organization (IOL). Statistics on working children and hazardous child labour in brief. Geneva: International Labour Organization; 1998. 
76. Alessi NP, Navarro VL. Health and work in rural areas: sugar cane plantation workers in Ribeirao Preto, Sao Paulo, Brazil. Cad. Saúde Pública. 1997;13 Suppl 2:111-121

77. Phyllis RC. Children, Tractor accidents, preventive measures. Agricultural Safety, 1997;42(12):18-23.

78. White L, O'Donnell C. Working children and accidents: Understanding the risks. Child Care Health Development. 2001; 27: 23-34.

79. Tucker L. Fingers to the bone: United States failure to protect child farmworkers, Washington DC: Human Rights Watch; 2000.

80. Schulman M, Evensen C, Runuyan C, Cohen L., Dunn K. Farm work is dangerous for teens: Agricultural hazards and Injuries among North Carolina Teens. Journal of Rural Health, 1997;(13): 295-305.

81. Schulman MD, Evensen CT, Runyan CW, Cohen LR, Dunn KA. Hearing loss among high school farm students. American Journal of Public Health. 1989;(79):619-622.

82. Banerjee SR. Child Labour in India: Present status (editorial). Indian Pediatr. 1995;32(4):403-408.

83. Belville R, Pollack SH, Godbold JH, Landrigan PJ. Occupational injuries among working adolescents in New York State. JAMA. 1993;269(21):2754-2759.

84. Layne LA, Castillo DN, Stout N, Cutlip P. Adolescent occupational injuries requiring hospital emergency department treatment: a nationally representative sample. Am. J. Public. Health. 1994;84(4):657-660.

85. Castillo DN, Landen D, Layne L. Occupational injury deaths of 16- and 17-yearolds in the United States. Am. J. Public. Health. 1994;84(4):646-649.

86. Windau J, Sygnatur E, Toscano G. Profile of work injuries incurred by young workers. Monthly Labor Review. 1999;122(6): 3-10.

87. Rosenman KD, Sims A, Luo Z, Gardiner J. Occurrence of lead-related symptoms below the current occupational safety and health act allowable blood lead levels. J. Occup. Environ. Med. 2003;45(5):546-555.

88. Reigart JR, Roberts JR. Pesticides in children. Pediatr. Clin. North Am. 2001 ;48(5):1185-1198.

89. Banerjee SR. Female working children. Indian Pediatr. 1990;27(11):1153-1158.

90. Chang TY, Jain RM, Wang CS, Chan CC. Effects of occupational noise exposure on blood pressure. J. Occup. Environ Med. 2003; 45(12):1289-1296.

91. Reijula K, Haahtela T, Klaukka T, Rantanen J. Incidence of occupational asthma and persistent asthma in young adults has increased in Findland. Chest. 1996;110(1): 58-61.

92. Burkhart G, Schulte PA, Robinson C, Sieber WK, Vossenas P, Ringen K. Job tasks, potential exposures, and health risks of laborers employed in the construction industry. Am. J. Ind. Med. 1993;24(4):413-425.

93. Kisner SM, Fosbroke DE. Injury hazards in the construction industry. J. Occup. Med. 1994;36(2):137-143.

94. Chavarría LV, Sánchez LN. Actividades laborales peligrosas e insalubres para la persona adolescente trabajadora. Costa rica: Consejo de Salud Ocupacional de Costa Rica. Área de Formación, Divulgación y Promoción; 2003. 
95. Bureau of International Labour Affairs. By the sweat and toil of children. Vol. 5: Efforts to eliminate child labour. Washington, DC:US Department of Labour; 1998.

96. Jensen RS. Press operator amputations: Is risk associated with age and gender? Journal of Safety Research. 1998;19:125-133.

97. Pollack SH, Landrigan PJ, Mallino DL. Child labor in 1990: prevalence and health hazards. Annu. Rev. Public Health. 1990;11:359-375.

98. Mitra S. A study of the health conditions of child workers in a small scale leather industry in Calcutta. Br. J. Ind. Med. 1993;50(10):938-940.

99. Thomas PT Zelikoff JT. Air Pollutans: Modulators of Pulmonary host resistance against infetions. Air Pollutions and Health. New York:Academic Press; 1999.

100. Romieu I, Samet JM, Smith KR, Bruce N. Outdoor air pollution and acute respiratory infections among children in developing countries. J. Occup. Environ. Med. 2002;44(7):640-649. 